\title{
Intracerebral Hemorrhage Caused by Cerebral Hyperperfusion after Superficial Temporal Artery to Middle Cerebral Artery Bypass for Atherosclerotic Occlusive Cerebrovascular Disease
}

\author{
Fumihiro Matano, ${ }_{1}$ Yasuo Murai, ${ }_{1}^{1}$ Takayuki Mizunari, ${ }^{2}$ Koji Adachi, ${ }^{3}$ Shiro Kobayashi, ${ }^{2}$ and Akio Morita ${ }^{1}$
}

Few papers have reported detailed accounts of intracerebral hemorrhage caused by cerebral hyperperfusion after superficial temporal artery to middle cerebral artery bypass (STA-MCA) bypass for atherosclerotic occlusive cerebrovascular disease. We report a case of vasogenic edema and subsequent intracerebral hemorrhage caused by the cerebral hyperperfusion syndrome (CHS) after STA-MCA bypass for atherosclerotic occlusive cerebrovascular disease disease without intense postoperative blood pressure control. A 63-year-old man with repeating left hemiparesis underwent magnetic resonance angiography (MRA), which revealed right internal carotid artery (ICA) occlusion. We performed a double bypass superficial temporal artery (STA)-middle cerebral artery (MCA) bypass surgery for the M2 and M3 branches. While the patient's postoperative course was relatively uneventful, he suffered generalized convulsions, and computed tomography revealed a low area in the right frontal lobe on Day 4 after surgery. We considered this lesion to be pure vasogenic edema caused by cerebral hyperperfusion after revascularization. Intravenous drip infusion of a free radical scavenger (edaravone) and efforts to reduce systolic blood pressure to $<120 \mathrm{mmHg}$ were continued. The patient experienced severe left hemiparesis and disturbance of consciousness on Day 8 after surgery, due to intracerebral hemorrhage in the right frontal lobe at the site of the earlier vasogenic edema. Brain edema associated with cerebral hyperperfusion after STA-MCA bypass for atherosclerotic occlusive cerebrovascular disease should be recognized as a risk factor for intracerebral hemorrhage. The development of brain edema associated with CHS after STA-MCA bypass for atherosclerotic occlusive cerebrovascular disease requires not only intensive control of blood pressure, but also consideration of sedation therapy with propofol.

Keywords: atherosclerotic; cerebral hyperperfusion; intracerebral hemorrhage; extracranial-intracranial bypass

\footnotetext{
${ }^{1}$ Department of Neurological Surgery, Nippon Medical School, Tokyo, Japan

${ }^{2}$ Department of Neurosurgery, Chiba Hokusoh Hospital, Chiba, Japan

${ }^{3}$ Department of Neurosurgery, Nippon Medical School Musashi Kosugi Hospital, Kanagawa, Japan
}

Received: February 15, 2016; Accepted: July 10, 2016

\section{Introduction}

Superficial temporal artery to middle cerebral artery (STA-MCA) bypass surgery for cerebrovascular ischemia, such as that caused by moyamoya disease and atherosclerotic occlusive disease was effective in improving blood flow to the brain to prevent stroke. ${ }^{1-3)}$ However, vascular reconstructive surgery may cause a rapid increase in cerebral blood flow, resulting in cerebral hyperperfusion syndrome (CHS).

Previous studies have reported that CHS is more frequent after STA-MCA bypass for moyamoya disease than after that for atherosclerotic disease, ${ }^{4)}$ and intracerebral hemorrhage ( $\mathrm{ICH}$ ) caused by CHS after STA-MCA bypass for moyamoya disease has also been reported. ${ }^{5-7)}$ In contrast, few studies have reported ICH caused by CHS after STA-MCA bypass for atherosclerotic occlusive cerebrovascular disease. Here, we describe a case of ICH caused by CHS after STA-MCA bypass for atherosclerotic occlusive cerebrovascular disease and review the related literature on this topic.

\section{Case Report}

A 63-year-old man with a history of hypertension presented at our hospital with repeated left hemiparesis. While a neurological examination at our facility revealed no abnormalities, magnetic resonance angiography (MRA) revealed right internal carotid artery occlusion (Fig. 1A). A singlephoton emission computed tomography (SPECT) scan, with and without acetazolamide challenge, revealed a perfusion defect in the right frontal lobe (hypoperfusion) and decreased cerebrovascular reactivity in the right hemisphere (Fig. 2). Perfusion computed tomography (CT) revealed hypoperfusion in the right frontal lobe (Fig. 3A).

We performed STA-MCA bypass surgery. Briefly, we dissected the STA during a craniotomy, and after incising through the dura mater and opening the Sylvian fissure, we selected an appropriate recipient segment, performing a double bypass for the M2 and M3 branches. Intra-operative trapping time was $27 \mathrm{~min}$ and $22 \mathrm{~min}$. The patient's postoperative course was relatively uneventful, and CT revealed no abnormalities (Fig. 4A). However, the patient suffered generalized convulsions, and CT revealed a low-density area in the right frontal lobe on Day 4 after surgery (Fig. 4B). On Day 5 after surgery, MRA revealed good bypass patency (Fig. 1B); magnetic resonance imaging (MRI) revealed high 


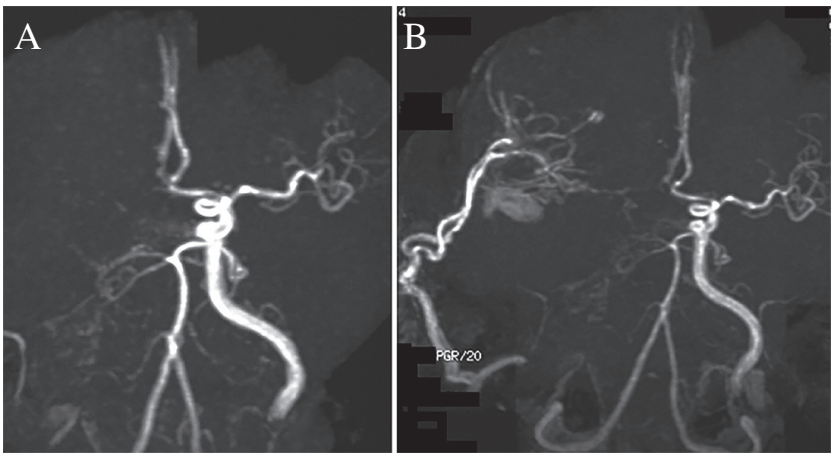

Fig. 1 (A) Pre-operative magnetic resonance angiography (MRA) revealed right internal carotid artery (ICA) occlusion. (B) Postoperative MRA revealed good bypass patency. intensity when $\mathrm{T}_{2}$-weighted (Fig. 5A) and slightly higher intensity when diffusion-weighted (Fig. 5B); and perfusion CT revealed hyperperfusion (Fig. 3B). We considered this lesion to be pure vasogenic edema caused by cerebral hyperperfusion after revascularization. Intravenous drip infusion of a free radical scavenger (edaravone) was continued, and systolic blood pressure was kept below $120 \mathrm{mmHg}$. The patient continued to experience severe left hemiparesis and disturbance of consciousness on Day 8 after surgery, due to intracerebral hemorrhage in the right frontal lobe at the site of the earlier vasogenic edema (Fig. 4C). We stopped anti-platelet therapy and continued to reduce systolic blood pressure. Follow up CT and MRI revealed no expanding hematoma or edema. The patient was transferred to another hospital for rehabilitation.

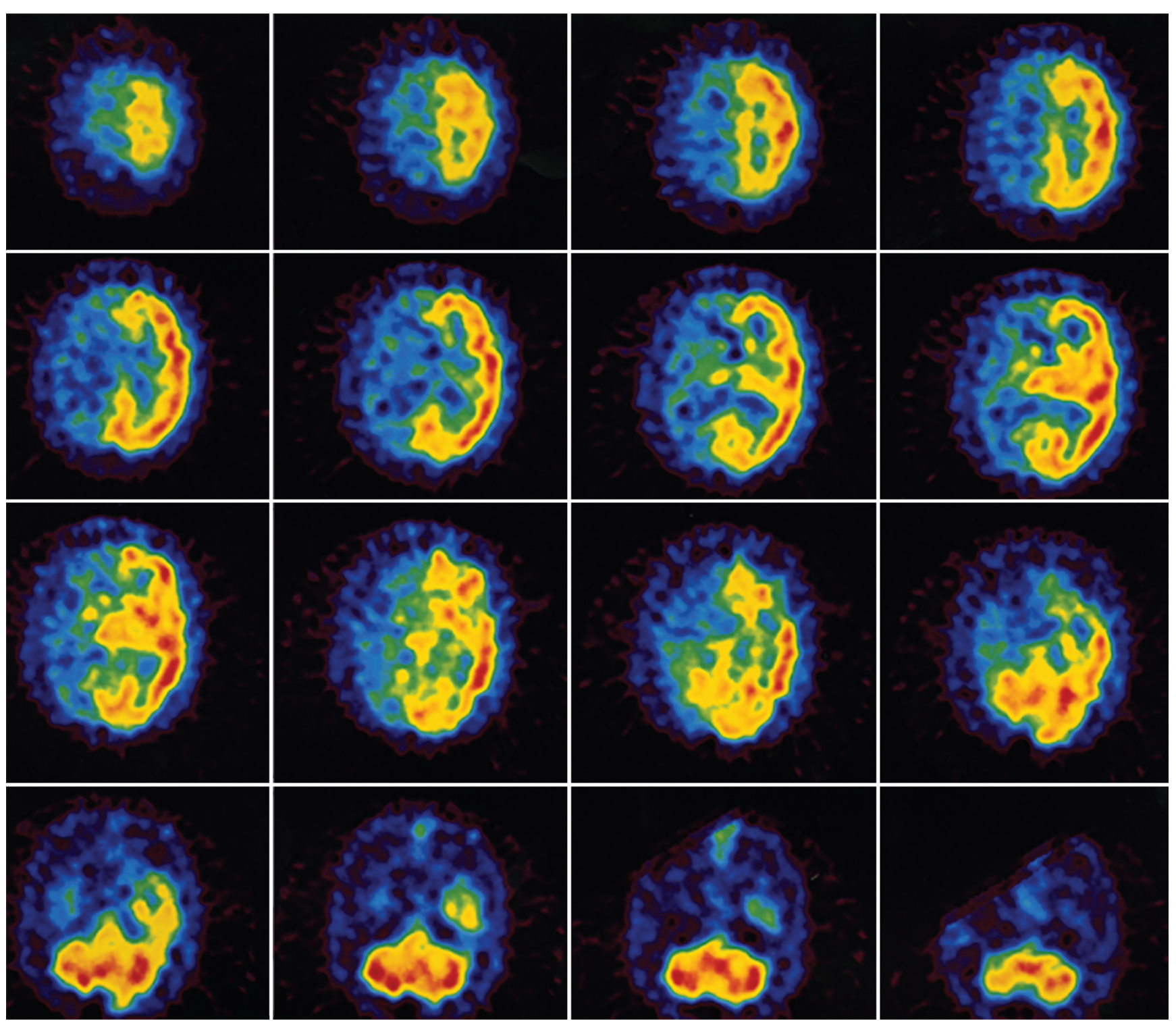

Fig. 2 Single-photon emission computed tomography (SPECT) scan revealed a perfusion defect in the right frontal (hypoperfusion) and decreased cerebrovascular reactivity in the right hemisphere on the pre-operative with acetazolamide challenge test. 

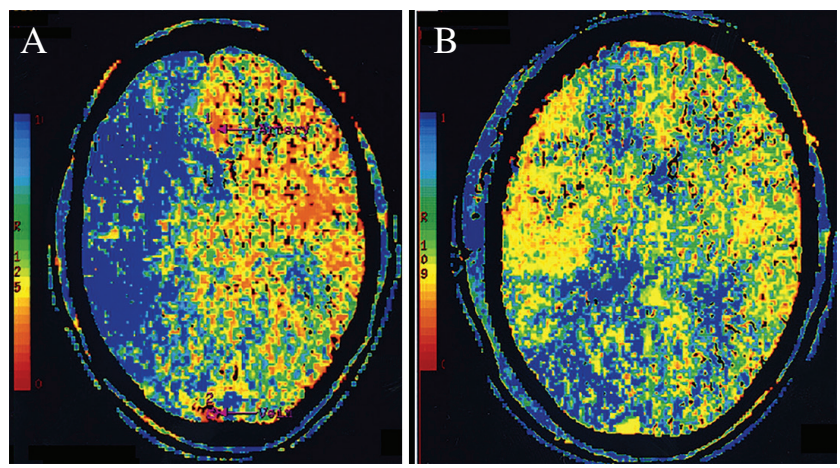

Fig. 3 (A) Pre-operative perfusion CT revealed at resting state revealed a perfusion defect (hypoperfusion) in the right frontal area of the right hemisphere. (B) Perfusion CT revealed hyperperfusion on Day 5 after surgery.

\section{Discussion}

We report a case of vasogenic edema and subsequent intracerebral hemorrhage caused by CHS after STA-MCA bypass for atherosclerotic occlusive cerebrovascular disease without intense postoperative blood pressure control. Brain edema associated with CHS after STA-MCA bypass for atherosclerotic occlusive cerebrovascular disease should be recognized as a risk factor of $\mathrm{ICH}$.

Vascular reconstruction surgery for patients with intracranial hypoperfusion can cause a rapid increase in cerebral blood flow, thereby resulting in CHS, which is characterized by unilateral headache, face and eye pain, seizures, and focal symptoms related to cerebral edema or intracranial hemorrhage. ${ }^{8,9)}$

Several methods of vascular reconstruction have been developed to treat ischemic cerebrovascular diseases, ${ }^{10,11)}$ and the frequency of hyperperfusion varies with each, ${ }^{9,12-18)}$ CHS

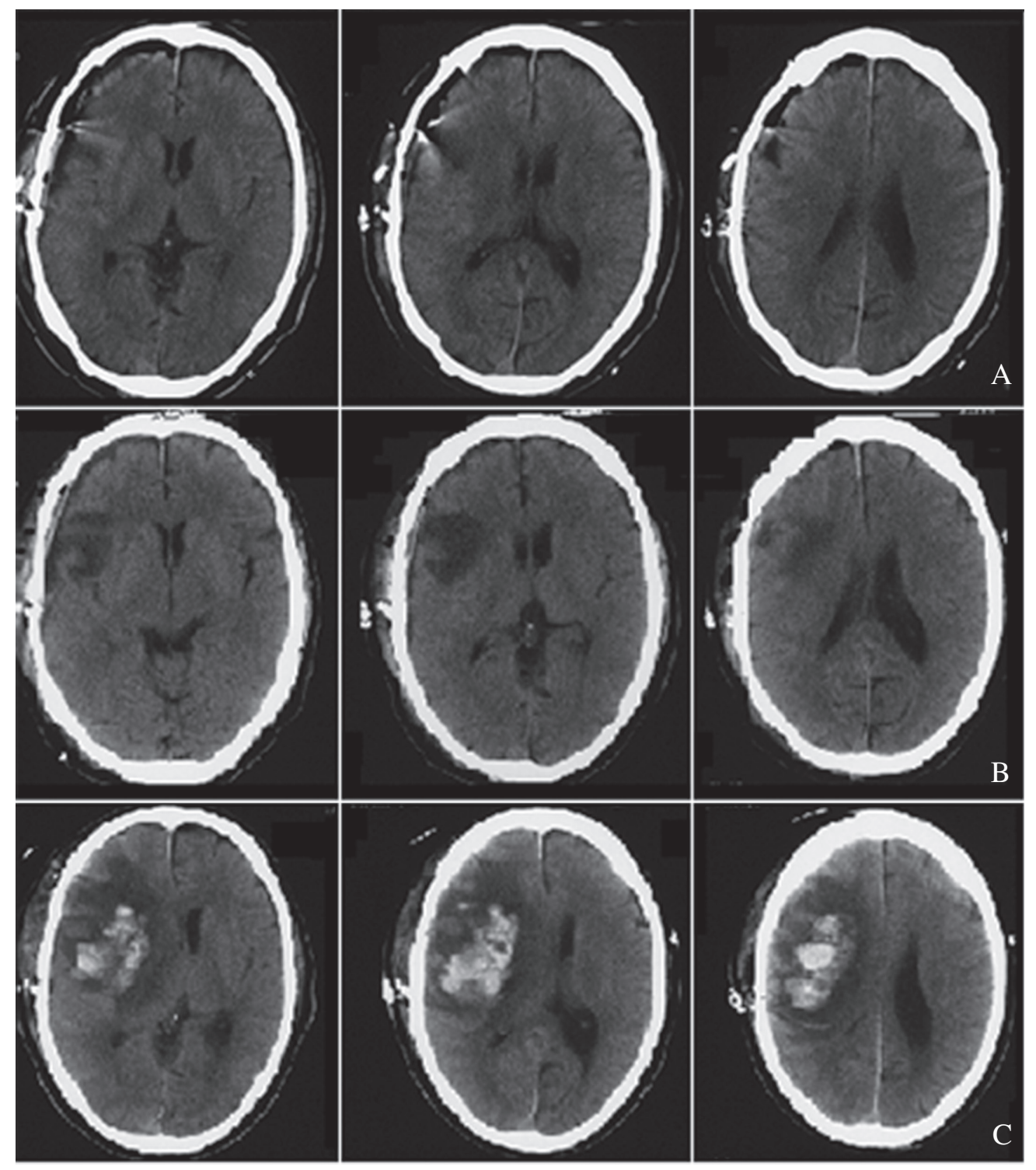

Fig. 4 (A) CT revealed no abnormalities on Day 1 after surgery. (B) CT revealed a low area in a right frontal on Day 4 after surgery. (C) CT revealed the right frontal intracerebral hemorrhage at the corresponding lesion to the prior vasogenic edema on Day 8 after surgery. 

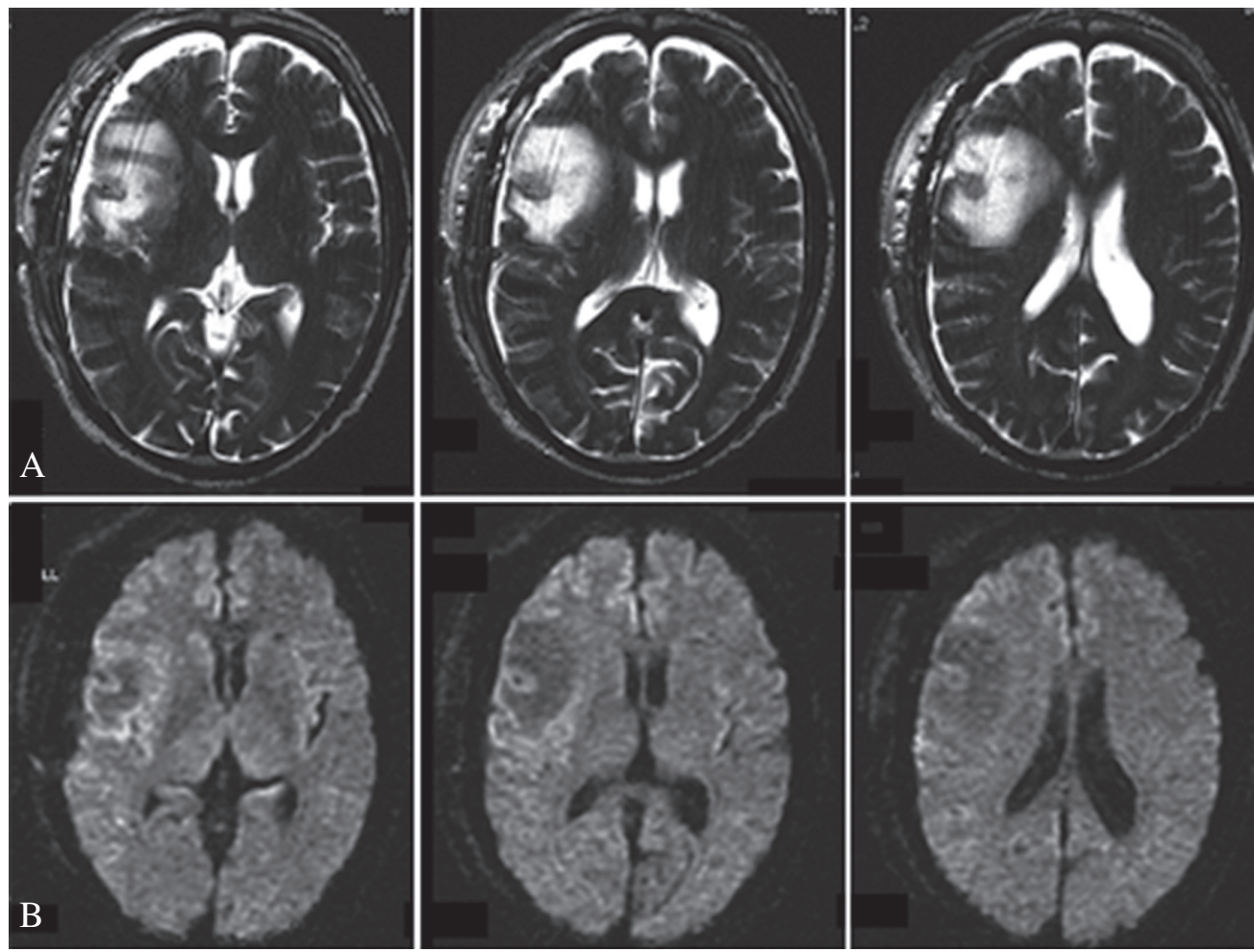

Fig. 5 (A) Magnetic resonance imaging (MRI) revealed the high intensity on $\mathrm{T}_{2}$-weight on the day after Day 5 after surgery. (B) Magnetic resonance imaging (MRI) revealed slightly high on diffusion on the day after Day 5 after surgery. and $\mathrm{ICH}$ caused by $\mathrm{CHS}$ occasionally occur in patients after carotid endarterectomy (CEA), carotid artery stenting (CAS), and STA-MCA bypass for moyamoya disease, , $9,14,17,19-21)^{\text {but }}$ CHS after STA-MCA bypass for atherosclerotic occlusive cerebrovascular disease is relatively rare $^{22-25)}$ and typically manifests as mild focal neurological deficit. Further, no detailed reports have been published on intracerebral hemorrhage caused by cerebral hyperperfusion after revascularization for atherosclerotic occlusive cerebrovascular disease.

Patients with poorer cerebrovascular reactivity and weakness of the blood-brain barrier due to chronic ischemia are known to have a potentially higher risk of hyperperfusion syndrome (HPS) ${ }^{20,26,27)}$ The increase of vascular endothelial growth factor and poorer network formation between the pial arteries in moyamoya disease contributes to the vulnerability to CHS in moyamoya patients compared to those with atherosclerotic disease. ${ }^{28,29)}$ In the present case, MRA revealed right ICA occlusion, and a SPECT scan showed an extensive area of hypoperfusion and decreased cerebrovascular reactivity in the right hemisphere. Given the above and the fact that we performed a double bypass for the M2 and M3 branches and not the M4 of cortical branch, the area of poorer cerebrovascular reactivity would receive a greater blood supply than is usual after bypass.

It may have caused the HPS and ICH observed in the present case. In fact, a previous report showed that STA-M4 bypass seldom resulted in post-operative HPS ${ }^{4)}$ Therefore, double anastomoses and M2 or M3 bypass may induce excessive flow and single bypass or M4 bypass should be appropriate.

Edema associated with HPS in the early stage is considered to be vasogenic edema. ${ }^{30-32)}$
Typical vasogenic edema appears as an area of low signal intensity on diffusion-weighted MRI. ${ }^{33)}$ In our case, when hyperperfusion occurred, the right frontal lobe at the site of anastomosis appeared as a low intensity lesion on CT, as a high-intensity area on $\mathrm{T}_{2}$-weighted MRI, and as a lowintensity area on diffusion-weighted MRI, findings which were typical of vasogenic edema.

Fujimura et al. ${ }^{34)}$ reported that a patient with moyamoya disease who underwent STA-MCA developed vasogenic edema due to HPS. Despite the patient's blood pressure being strictly controlled to less than $120 \mathrm{mmHg}$, the edema progressed to intracerebral hemorrhage. These authors concluded that an early increase in cerebral blood flow associated with vasogenic edema formation at the site of anastomosis may be a warning sign for subsequent hemorrhagic complications. We observed a similar clinical course with the present case and drew a similar conclusion regarding STA-MCA bypass for atherosclerotic occlusive cerebrovascular disease. In our case, we controlled blood pressure to less than $140 \mathrm{mmHg}$ from immediately after operation to the development of vasogenic edema, but this resulted in the development of critical vasogenic edema. Intensive anti-hypertensive treatment (systolic blood pressure $<130 \mathrm{mmHg}$ ) immediately after STA-MCA bypass surgery prevents HPS in moyamoya disease, ${ }^{35)}$ suggesting the need for strict postoperative control of blood pressure in atherosclerotic occlusive cerebrovascular disease.

Seizure due to HPS has been reported as a contraindication for anticoagulation therapy due to the increased risk of hemorrhage in such patients. ${ }^{9)}$ Continuous sedation is therefore recommended for the first $48 \mathrm{~h}$ post-surgery to prevent hyperperfusion. ${ }^{36)}$ 
Although these studies targeted CEA, we should consider the use of propofol sedation therapy, which reduces lactate production and cerebral metabolic rate ${ }^{37)}$ when brain edema associated with CHS appears. ${ }^{36,38)}$

\section{Conclusion}

We report a case of vasogenic edema following intracerebral hemorrhage caused by CHS after STA-MCA bypass for atherosclerotic occlusive cerebrovascular disease without intense postoperative blood pressure control. The use of propofol sedation therapy to prevent ICH due to CHS should be considered in patients developing vasogenic edema.

\section{Conflicts of Interest Disclosure}

The authors have no conflicts of interest. All authors who are members of the Japan Neurosurgical Society (JNS) have registered on line Self-reported COI Disclosure Statement Forms through the website for JNS members.

\section{References}

1) Fluri F, Engelter S, Lyrer P: Extracranial-intracranial arterial bypass surgery for occlusive carotid artery disease. Cochrane Database Syst Rev CD005953, 2010

2) Holohan TV: Extracranial-intracranial bypass to reduce the risk of ischemic stroke. CMAJ 144: 1457-1465, 1991

3) Murai Y, Mizunari T, Takagi R, Amano Y, Mizumura S, Komaba Y, Okubo S, Kobayashi S, Teramoto A: Analysis of ischemic cerebral lesions using 3.0-T diffusion-weighted imaging and magnetic resonance angiography after revascularization surgery for ischemic disease. Clin Neurol Neurosurg 115: 1063-1070, 2013

4) Fujimura M, Shimizu H, Inoue T, Mugikura S, Saito A, Tominaga T: Significance of focal cerebral hyperperfusion as a cause of transient neurologic deterioration after extracranial-intracranial bypass for moyamoya disease: comparative study with non-moyamoya patients using $\mathrm{N}$-isopropyl-p-[(123)I]iodoamphetamine single-photon emission computed tomography. Neurosurgery 68: 957-964, 2011

5) Hosoda K, Kawaguchi T, Ishii K, Minoshima S, Shibata Y, Iwakura M, Ishiguro S, Kohmura E: Prediction of hyperperfusion after carotid endarterectomy by brain SPECT analysis with semiquantitative statistical mapping method. Stroke 34: 1187-1193, 2003

6) Narisawa A, Fujimura M, Shimizu H and Tominaga T: [Seizure following superficial temporal-middle cerebral artery anastomosis in patients with moyamoya disease: possible contribution of postoperative cerebral hyperperfusion]. No Shinkei Geka 35: 467-474, 2007

7) Fujimura M, Mugikura S, Kaneta T, Shimizu H, Tominaga T: Incidence and risk factors for symptomatic cerebral hyperperfusion after superficial temporal artery-middle cerebral artery anastomosis in patients with moyamoya disease. Surg Neurol 71: 442-447, 2009

8) Sundt TM, Sandok BA, Whisnant JP: Carotid endarterectomy. complications and preoperative assessment of risk. Mayo Clin Proc 50: $301-$ 306, 1975

9) Piepgras DG, Morgan MK, Sundt TM, Yanagihara T, Mussman LM: Intracerebral hemorrhage after carotid endarterectomy. J Neurosurg 68: 532-536, 1988

10) Gröschel K, Riecker A, Schulz JB, Ernemann U, Kastrup A: Systematic review of early recurrent stenosis after carotid angioplasty and stenting. Stroke 36: 367-373, 2005

11) Halliday A, Mansfield A, Marro J, Peto C, Peto R, Potter J, Thomas D; MRC Asymptomatic Carotid Surgery Trial (ACST) Collaborative Group: Prevention of disabling and fatal strokes by successful carotid endarterectomy in patients without recent neurological symptoms: randomised controlled trial. Lancet 363: 1491-1502, 2004

12) Katano H, Mase M, Sakurai K, Miyachi S, Yamada K: Revaluation of collateral pathways as escape routes from hyperemia/hyperperfusion following surgical treatment for carotid stenosis. Acta Neurochir (Wien) 154: 2139-2148, 2012

13) Yoshimura S, Kitajima H, Enomoto Y, Yamada K, Iwama T: Staged angioplasty for carotid artery stenosis to prevent postoperative hyperperfusion. Neurosurgery 64: ons122-ons128; discussion ons128-ons129, 2009
14) Sundt TM, Sharbrough FW, Piepgras DG, Kearns TP, Messick JM, O'Fallon WM: Correlation of cerebral blood flow and electroencephalographic changes during carotid endarterectomy: with results of surgery and hemodynamics of cerebral ischemia. Mayo Clin Proc 56: 533-543, 1981

15) Ogasawara K, Sakai N, Kuroiwa T, Hosoda K, Iihara K, Toyoda K, Sakai C, Nagata I, Ogawa A; Japanese Society for Treatment at Neck in Cerebrovascular Disease Study Group: Intracranial hemorrhage associated with cerebral hyperperfusion syndrome following carotid endarterectomy and carotid artery stenting: retrospective review of 4494 patients. J Neurosurg 107: 1130-1136, 2007

16) Hafner DH, Smith RB, King OW, Perdue GD, Stewart MT, Rosenthal D, Jordan WD: Massive intracerebral hemorrhage following carotid endarterectomy. Arch Surg 122: 305-307, 1987

17) Solomon RA, Loftus CM, Quest DO, Correll JW: Incidence and etiology of intracerebral hemorrhage following carotid endarterectomy. J Neurosurg 64: 29-34, 1986

18) Abou-Chebl A, Yadav JS, Reginelli JP, Bajzer C, Bhatt D, Krieger DW: Intracranial hemorrhage and hyperperfusion syndrome following carotid artery stenting: risk factors, prevention, and treatment. J Am Coll Cardiol 43: 1596-1601, 2004

19) Wu TY, Anderson NE, Barber PA: Neurological complications of carotid revascularisation. J Neurol Neurosurg Psychiatry 83: 543-550, 2012

20) van Mook WN, Rennenberg RJ, Schurink GW, van Oostenbrugge RJ, Mess WH, Hofman PA, de Leeuw PW: Cerebral hyperperfusion syndrome. Lancet Neurol 4: 877-888, 2005

21) Ohue S, Kumon Y, Kohno K, Watanabe H, Iwata S, Ohnishi T: Postoperative temporary neurological deficits in adults with moyamoya disease. Surg Neurol 69: 281-286; discussion 286-287, 2008

22) Heros RC, Scott RM, Kistler JP, Ackerman RH, Conner ES: Temporary neurological deterioration after extracranial-intracranial bypass. Neurosurgery 15: 178-185, 1984

23) Higashi S, Matsuda H, Fujii H, Ito H, Yamashita J: Luxury perfusion syndrome confirmed by sequential studies of regional cerebral blood flow and volume after extracranial to intracranial bypass surgery: case report. Neurosurgery 25: 85-89, 1989

24) Kuroda S, Kamiyama H, Abe H, Asaoka K, Mitsumori K: Temporary neurological deterioration caused by hyperperfusion after extracranialintracranial bypass - case report and study of cerebral hemodynamics. Neurol Med Chir (Tokyo) 34: 15-19, 1994

25) Uno M, Nakajima N, Nishi K, Shinno K, Nagahiro S: Hyperperfusion syndrome after extracranial-intracranial bypass in a patient with moyamoya disease_-case report. Neurol Med Chir (Tokyo) 38: 420_ 424, 1998

26) Ogasawara K, Yukawa H, Kobayashi M, Mikami C, Konno H, Terasaki $\mathrm{K}$, Inoue T, Ogawa A: Prediction and monitoring of cerebral hyperperfusion after carotid endarterectomy by using single-photon emission computerized tomography scanning. J Neurosurg 99: 504-510, 2003

27) Yamaguchi K, Kawamata T, Kawashima A, Hori T, Okada Y: Incidence and predictive factors of cerebral hyperperfusion after extracranial-intracranial bypass for occlusive cerebrovascular diseases. Neurosurgery 67: 1548-1554; discussion 1554, 2010

28) Kim JE, Oh CW, Kwon OK, Park SQ, Kim SE, Kim YK: Transient hyperperfusion after superficial temporal artery/middle cerebral artery bypass surgery as a possible cause of postoperative transient neurological deterioration. Cerebrovasc Dis 25: 580-586, 2008

29) Fujimura M, Watanabe M, Narisawa A, Shimizu H, Tominaga T: Increased expression of serum Matrix Metalloproteinase-9 in patients with moyamoya disease. Surg Neurol 72: 476-480; discussion 480, 2009

30) Ay H, Buonanno FS, Schaefer PW, Le DA, Wang B, Gonzalez RG, Koroshetz WJ: Posterior leukoencephalopathy without severe hypertension: utility of diffusion-weighted MRI. Neurology 51: 1369-1376, 1998

31) Casey SO, Truwit CL: Pontine reversible edema: a newly recognized imaging variant of hypertensive encephalopathy? AJNR Am J Neuroradiol 21: 243-245, 2000

32) Covarrubias DJ, Luetmer PH, Campeau NG: Posterior reversible encephalopathy syndrome: prognostic utility of quantitative diffusion-weighted MR images. AJNR Am J Neuroradiol 23: 1038-1048, 2002

33) Kokuzawa J, Kaku Y, Watarai T, Tanaka T, Hatsuda N, Ando T: Pure vasogenic edema caused by cerebral hyperperfusion after superficial 
temporal artery to middle cerebral artery anastomosis-case report. Neurol Med Chir (Tokyo) 50: 250-253, 2010

34) Fujimura M, Shimizu H, Mugikura $S$, Tominaga T: Delayed intracerebral hemorrhage after superficial temporal artery-middle cerebral artery anastomosis in a patient with moyamoya disease: possible involvement of cerebral hyperperfusion and increased vascular permeability. Surg Neurol 71: 223-227; discussion 227, 2009

35) Fujimura M, Inoue T, Shimizu H, Saito A, Mugikura S, Tominaga T: Efficacy of prophylactic blood pressure lowering according to a standardized postoperative management protocol to prevent symptomatic cerebral hyperperfusion after direct revascularization surgery for moyamoya disease. Cerebrovasc Dis 33: 436-445, 2012
36) Kawamata T, Okada Y, Kawashima A, Yoneyama T, Yamaguchi K Ono Y, Hori T: Postcarotid endarterectomy cerebral hyperperfusion can be prevented by minimizing intraoperative cerebral ischemia and strict postoperative blood pressure control under continuous sedation. Neurosurgery 64: 447-453, 2009

37) Kalimeris K, Kouni S, Kostopanagiotou G, Nomikos T, Fragopoulou E, Kakisis J, Vasdekis S, Matsota P, Pandazi A: Cognitive function and oxidative stress after carotid endarterectomy: comparison of propofol to sevoflurane anesthesia. J Cardiothorac Vasc Anesth 27: 1246-1252, 2013

38) Lao N, Nie H, Xu LX, Xiong LZ, Zhang H, Fan YY, Wang BR: Efficacy of intracarotid propofol infusion and impact of cerebral blood flow alteration. Br J Anaesth 102: 234-239, 2009

Corresponding author:

Fumihiro Matano, Department of Neurosurgery, Chiba Hokusoh Hospital, 1715 Kamagari, Inzai, Chiba, 270-1694, Japan.

$\llbracket$ s00-078@nms.ac.jp 